\title{
Analysis of a clinical case on hysterocyesis combining with caesarean scar pregnancy after in vitro fertilization-embryo transfer
}

\author{
Liu $X^{1}$, Li D ${ }^{2}$, Wang $Q^{1}$, Ren $C^{3,4}$ and Liu $W^{*}$ \\ ${ }^{1}$ Qingdao Women and Children's Hospital, NO.6 Tongfu Road, Qingdao City, Shandong Province, 266034, China \\ ${ }^{2}$ Qingdao Center Hospital, NO.127 Siliunan Road, Qingdao City, Shandong Province, 266031, China \\ ${ }^{3}$ Affiliated Hospital of Weifang Medical University, NO.2428 Yuhe Road, Weifang City, Shandong Province, 261031, China \\ ${ }^{4}$ Key Laboratory of Reproductive Medicine of Shandong Province Health Department, NO.2428 Yuhe Road, Weifang City, Shandong Province, 261031, China
}

\begin{abstract}
Aim: To explore safe and effective therapeutic strategies for hysterocyesis concurrency of caesarean scar pregnancy (CSP) that will be able to remedy the foetus within uterus.

Study design: Repeated ultrasound scans confirmed hysterocyesis concurrency of CSP in a patient that conceived by in vitro fertilization-embryo transfer (IVF-ET) in our department on the $35^{\text {th }}$ day of embryo transfer. After being fully informed and signing consent, selective embryo reduction that implanted on the caesarean scar by needle aspiration of the embryo via transvaginal ultrasonography was performed. Transvaginal ultrasonography reexamination was performed on $7^{\text {th }}$ day after operation. Anti-miscarriage treatments and regular obstetric check-up ensued.
\end{abstract}

Results: Emergency caesarean section was operated on $38^{\text {th }}$ week of gestation and a live infant was delivered and appeared developmentally normal. There was no heavy intraoperative haemorrhage and metrorrhexis symptom observed in the operation. Mater and infant were doing well.

Conclusion: Hysterocyesis complicated CSP after IVF-ET is extremely rare. After early diagnosis, ultrasonic inducing needle aspiration of the embryo implanted on the caesarean scar can terminate CSP while hysterocyesis is effectively saving. This treatment can receive a satisfactory clinical outcome.

\section{Case report}

A 34-year-old woman, $\mathrm{G}_{2} \mathrm{P}_{2} \mathrm{~L}_{1}$, underwent selective embryo reduction in our hospital on 8 May 2013 for an intrauterine pregnancy combined a caesarean scar pregnancy (CSP) after in vitro fertilizationembryo transfer (IVF-ET). She had a spontaneous pregnancy and underwent a lower uterine segment cesarean section for a breech presentation and non-reducible umbilical cord around the neck 7 years ago. She wore an intrauterine device for 4 years. Her child drowned, and she was subsequently diagnosed with secondary infertility (bilateral fallopian tube obstruction and mild hydrosalpinx). The woman underwent IVF-ET on 1 April 2013. Two embryos with good morphology were transferred into the uterus under abdominal ultrasound guidance. The result of the pregnancy test was positive 14 days after ET, and all further evaluations showed a regular $\beta$-hCG increment. A vaginal ultrasound examination performed at 35 days showed a gravid uterus with a $2.9 \times 2.1 \mathrm{~cm}$ intrauterine gestational sac with germ tube and beating heart. And another embryo implanted in the inferior portion of the uterus closed to the caesarean section scar with a $2.3 \times 1.7 \mathrm{~cm}$ gestational sac with germ tube and beating heart, where the thinnest site of the uterine wall was $0.25 \mathrm{~cm}$ (Figure 1). There were no abnormalities in the cervical and double accessories, and no free fluid in the pelvis. After an obstetrics consultation was obtained, the admission diagnosis was pregnancy after IVF-ET (two live embryos) with cesarean scar pregnancy (CSP). After explaining the pregnancy status to patient and her family, and in consideration of the viable intrauterine embryo, selective reduction of the embryo that implanted on the caesarean scar was performed after being fully informed and signing consent. On 8 May 2013, via transvaginal ultrasonography guidance, a $17 \mathrm{G}$ double-lumen needle punctured the germ tube of the gestational scar with a negative pressure suction $(120 \mathrm{mmHg})$ was applied to aspirate the cystic contents, blastocyst, and chorionic tissue. The gestational scar was then injected with $2 \mathrm{ml}$ of $50 \%$ glucose. Transvaginal ultrasonography reexamination didn't reveal intracystic hemorrhage and the beating heart in the gestational sac in the inferior part of the uterus was normal. The procedure was successful without significant bleeding. After 7 days, a transvaginal ultrasonography repeated confirmed a gravid uterus with a $3.4 \times 2.3 \mathrm{~cm}$ gestational sac, germ tube, and beating heart throb in the inferior part of the uterus and a $1.5 \times 1.1 \mathrm{~cm}$ medium with heterogeneous echo pattern, with absence of blood flow, germ tube, and beating heart in the lower segment of the anterior uterus. There were no abnormalities in the cervical, double accessories and free fluid in the pelvis (Figure 2). The patient was stable,

*Correspondence to: Weiqin Liu, Qingdao Women and Children's Hospital, NO.6 Tongfu Road, Qingdao City, Shandong Province 266034, China, E-mail: lxq_890@hotmail.com

Key words: in vitro fertilization-embryo transfer (ivf-et), cesarean scar pregnancy (csp) heterotopic pregnancy, selective embryo reduction

Received: March 22, 2018; Accepted: April 23, 2018; Published: April 26, 2018 


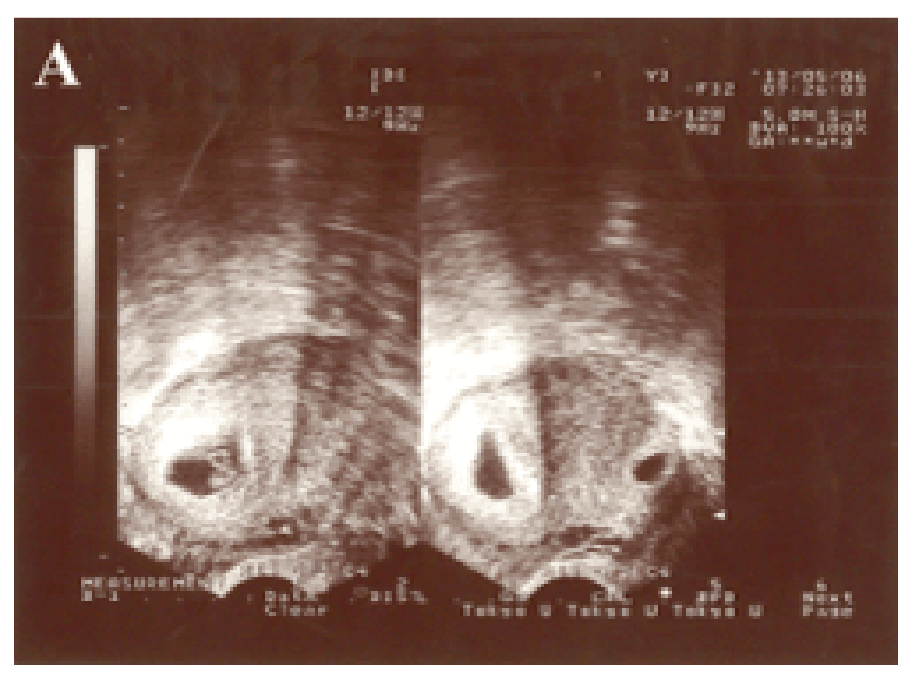

Figure 1. Transvaginal ultrasonography examination before selective embryo reduction. There were two gestational sacs in the uterine cavity, one (as shown the left one of the right portion) in the inferior portion of the uterus and the other (as shown the right one of the right portion) in the lower segment scar area of the anterior uterus, both with germ tubes and beating hearts

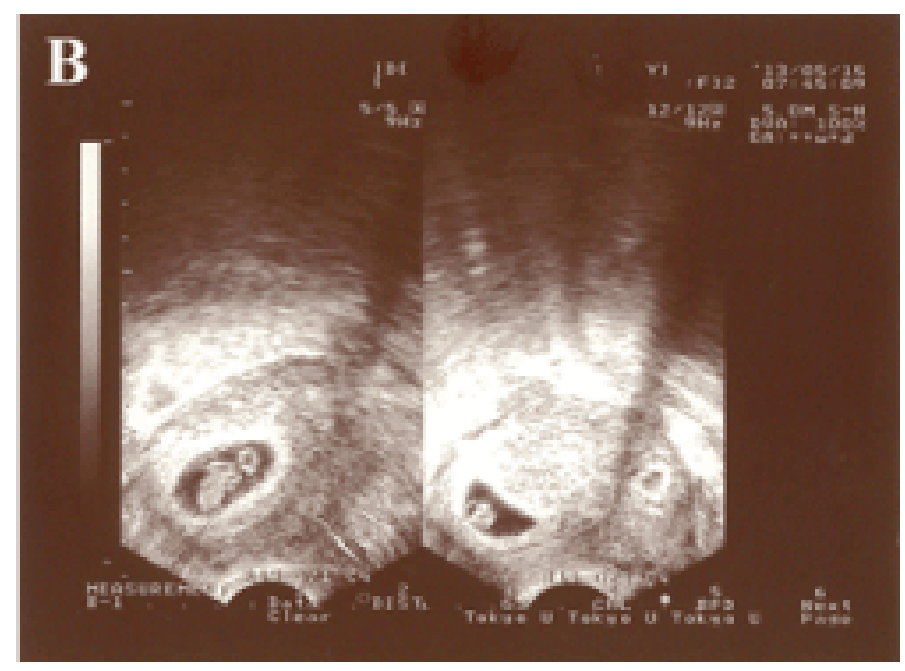

Figure 2. Selective embryo reduction followed by transvaginal ultrasonography examination after 7 days. The gestational sac (as shown the left one of the right portion) in the inferior portion of the uterus was increased, and the gestational sac (as shown the right one of the right portion) without a germ tube in the lower segment scar area of the anterior uterus was degenerated

and there was no abdominal pain, vaginal bleeding, or fluid discharge, so she was permitted to return home. Anti-miscarriage treatments and regular obstetric check-up ensued. Emergency caesarean section was operated in $38^{\text {th }}$ week of gestation and a live infant was delivered and appeared developmentally normal. There was no heavy intraoperative haemorrhage and metrorrhexis symptom observed in the operation and the placenta was located in the posterior uterine wall. Surgical exploration of the uterine scar evidenced no sign of rupture. Mater and infant were doing well.

\section{Discussion}

A CSP refers to a fertilized egg or placenta implantation in a previous uterine incision scar and is a rare type of ectopic pregnancy [1]. An intrauterine pregnancy in combination with an ectopic pregnancy is referred to as a heterotopic pregnancy (HP). While there is a small number of clinical reports involving HPs, an intrauterine pregnancy combined a CSP is less common, with a reported incidence of $1 / 2216$ to $1 / 1800$ in international reports, comprising $6.1 \%$ of ectopic pregnancie [2]

For the majority of HPs, the ectopic pregnancy occurs in the fallopian tubes. Both conservative and surgical treatments can yield a favorable therapeutic effect, and the intrauterine pregnancy can continue. When the ectopic pregnancy is located in the uterine incision of a previous cesarean section, serious complications may arise, such as uterine rupture and bleeding. As a result, the intrauterine pregnancy cannot continue, and the situation may become life-threatening if not managed in a timely fashion. Due to the low incidence of CSPs, there are no universal treatment guidelines. At present, the common treatments which have been adopted for CSPs mainly include curettage followed by uterine artery embolism, curettage under Doppler ultrasonography guidance, curettage followed by methotrexate therapy, laparoscopic capsule collection with suturing, open laparotomy with topical incision, local paracentesis, and subtotal hysterectomy or total hysterectomy [3]. Asymptomatic patients (no abdominal pain or vaginal bleeding) can be considered conservative therapy without unbefitting curettage. However, the clinical effects of conservative treatment is often not very satisfactory, which usually combines with curettage and lesion excision [4]. Patients with severe abdominal pain and heavy vaginal bleeding must be administered surgery in visual excision of the lesion and immediate repair of the scar to achieve a positive outcome [5]. In the absence of a hysterocyesis or for patients considering pregnancy termination, surgery and drug injection therapy can be performed. Previous reports have shown that when treated with combined therapy consisting of methotrexate and mifepristone, and then managed by surgery with hysteroscopy-laparoscopy on patients with CSPs, the gestational tissue can be removed and the uterine coloboma can be suture-repaired. These treatments could evaluate the presence of uterine bleeding and suture of local coloboma, preserve fertility and reduce the possibility of scar pregnancies in the same location [6]. Although conventional treatments can achieve satisfactory clinical outcomes, the purpose is to terminate pregnancy, which not only terminate scar pregnancies, but the intrauterine pregnancies that exist.

Hysterocyesis should be attached importance on patients with desired fertility, although the incidence of intrauterine pregnancy with CSPs caused by assisted reproductive technology is extremely low. Therefore, conservative medical and conventional surgery are bound to not work. Seeking a safe treatment method for both maternal and hysterocyesis is a key problem that we faced. This patient experienced a hysterocyesis with a CSP after IVF-ET and the intrauterine embryo was very precious to her and her family. The continued development of the intrauterine embryo and maternal safety must be assured. In the treatment, we were able to establish an early diagnosis of the CSP using transvaginal ultrasonography examination. Via transvaginal ultrasonography guidance, selective reduction of the embryo that implanted on the caesarean scar by needle aspiration was performed and glucose was injected successfully. The intrauterine pregnancy was carried to term and a satisfactory clinical outcome was achieved. In the treatment of simple CSPs and HPs, Doubilet et al [7] reported that under transvaginal ultrasonography guidance, 27 patients underwent selective embryo reduction by puncturing ectopic gestational sacs or embryos and injecting $\mathrm{KCl}$, which achieved good clinical therapeutic effects. Compared with conventional treatments, interference of selective embryo reduction on maternal trauma and intrauterine pregnancy were minimal. Therefore, after early diagnosis of patients with intrauterine pregnancies combining with CSPs, many scholars be inclined to perform selective embryo reduction $[8,9]$. These show that selective embryo reduction of puncture into the gestational sac in the lower segment of the uterus via the vagina is feasible. 
With the increased cesarean section rate and the application of assisted reproductive technology, the incidence of CSPs is likely to increase. The increased incidence of CSPs after IVF-ET may be related to transplantation of more embryos (2-3), a higher placed position of embryos, migration of the embryo, stimulation to the uterus by transplantation and uterine contractions that lead to a change in the embryo implantation position. To reduce the occurrence of CSPs or uterine rupture, the previous cesarean incision section should be fully evaluated by ultrasound or hysteroscopy before IVF-ET. In the process of embryo-transplantation, specific operative procedures and operation skills must be performed to prevent possible complications or avoid the appearance of CSPs. For CSPs, transvaginal sonography has obvious advantages as quick implementation, convenience and high diagnostic rate, which should be the choice of the most valuable examination method [10]. The standard for ultrasonic diagnosis of CSPs includes gestational sac trophoblast lying between the anterior wall of the uterine and bladder, absence of an intrauterine pregnancy sac sonographically, lacking continuity of the myometrium of the uterine anterior wall between the gestational sac and bladder on the sagittal plane and positive embryo sac sliding [11,12]. When patients present with hysterocyesis combined CSP after IVF-ET and have been diagnosed by transvaginal sonography, puncturing the gestational scar by needle and aspirating the embryo in the uterine lower segment scar area can achieve the purpose of scar pregnancy termination and have no negative effects on intrauterine pregnancy, which is a practical and effective method for preserving intrauterine pregnancy and treating CSP at the same time. Patients, as well as their families, should be fully informed about the potential risk and outcome before selective embryo reduction. And the medical staff should arrange strict follow-up and regular obstetric check-up after de-embryo operation with the purpose of ensuring the pregnancy on the rails and receiving a satisfactory clinical outcome.

\section{Acknowledgments}

The authors are sincerely indebted Professor Shiwen Jiang, Director of Epigenetic Laboratory at Mercer University School of Medicine, for his critiques and revision of the paper. This study was supported by Shandong Natural Science Fund (ZR2015HL020).

\section{Disclosure}

Xiaoqiang Liu and Dan Li are the co-first author. Weiqin Liu is the corresponding author.

\section{Conflict of interests}

The authors declare that there is no conflict of interests regarding the publication of this paper.

\section{References}

1. Timor-Tritsch IE, Monteagudo A, Santos R, et al. (2012) The diagnosis, treatment, and follow-up of cesarean scar pregnancy. Am J Obstet Gynecol 207: 44e1-e13. [Crossref]

2. Rotas MA, Haberman S, Levgur M (2006) Cesarean scar ectopic pregnancies: etiology, diagnosis, and management. Obstet Gynecol 107: 1373-81. [Crossref]

3. Family planning branch of the Chinese Medical Association. Diagnosis and treatment consensus of cesarean scar pregnancy. National Medical Journal of China 92: 17311733.

4. Yang XL, Zhou YF (2014) The conservative treatment of cesarean scar pregnancy. Journal of Practical Obstetrics and Gynecology 30: 252-253.

5. Lin AP, Yang Z, Jiang XW (2014) Exploring clinical classification and laparoscopic surgery of cesarean scar pregnancy. Journal of Practical Obstetrics and Gynecology 30: 266-269.

6. Luo YP, Wang YL, Yang L (2012) The hysteroscopy combined with laparoscopy diagnose and treat cesarean scar pregnancy: 8 cases of clinical analysis. Journal of Practical Obstetrics and Gynecology 28: 1070-1071.

7. Doubilet PM, Benson CB, Frates MC (2004) Sonographically guided minimally invasive treatment of unusual ectopic pregnancies. J Ultrasound Med 23: 359-370. [Crossref]

8. Litwicka K, Greco E, Prefumo F (2011) Successful management of a triplet heterotopic caesarean scar pregnancy after in vitro fertilization-embryo transfer. Fertil Steril 95 : 291.e1-e3. [Crossref]

9. Ugurlucan FG, Bastu E, Dogan M (2012) Management of cesarean heterotopic pregnancy with transvaginal ultrasound-guided potassium chloride injection and gestational sac aspiration, and review of the literature. J Minim Invasive Gynecol 19: 671-673. [Crossref]

10. Shao HJ, Ma JT, Yang XE (2010) Exploring the way of diagnosis and treatment of cesarean scar pregnancy. National Medical Journal of China 90: 2616-2619.

11. Vial Y, Petignat P, Hohlfeld P (2000) Pregnancy in a cesarean scar. Ultrasound Obstet Gynecol 16: 592-593. [Crossref]

12. Jurkovic D, Hillaby K, Woelfer B (2003) First-trimester diagnosis and management of pregnancies implanted into the lower uterine segment Cesarean section scar. Ultrasound Obstet Gynecol 21: 220-227. [Crossref]

Copyright: (C2018 Liu X. This is an open-access article distributed under the terms of the Creative Commons Attribution License, which permits unrestricted use, distribution, and reproduction in any medium, provided the original author and source are credited. 